\title{
SATISFACCIÓN DEL TRATO RECIBIDO DURANTE EL EMBARAZO, PARTO Y PUERPERIO EN UN HOSPITAL PÚBLICO
}

SATISFACTION REGARDING CARE RECEIVED DURING PREGNANCY, DELIVERY AND POSTPARTUM AT A PUBLIC HOSPITAL

María Sobeida Leticia Blazquez Morales ${ }^{1}$ Karen Lizeth MuñozAguilar $^{2}$

Gaudencio GutiérrezAlba $^{3}$ José Isaías SiliceoMurrieta $^{4}$

${ }^{1}$ Doctora en Educación, Médico Cirujano. Instituto de Ciencias de la Salud Universidad Veracruzana Xalapa, Veracruz, México. Email.: sobeida_blazquez@ hotmail.com

${ }^{2}$ Maestra en Ciencias de la Salud, Médico Cirujano. Instituto de Ciencias de la Salud Universidad Veracruzana en Xalapa Veracruz México

${ }^{3}$ Doctor en Ciencias de la Salud, Médico Cirujano . Instituto de Ciencias de la Salud Universidad Veracruzana en Xalapa Veracruz México ${ }^{4}$ Maestría en Salud Pública, División de Estudios de Posgrado, Universidad de la Sierra Sur, Oaxaca, México. Profesor Instructor, Escuela de Psicología, Universidad Adolfo Ibáñez, Santiago, Chile.

Trabajo recibido: 14 de Noviembre 2017 Aprobado: 27 de Febrero 2018.

\section{SATISFAÇÃO DO TRATAMENTO RECEBIDO DURANTE A GRAVIDEZ, PARTO E PUER- PÉRIO EM UM HOSPITAL PÚBLICO}

\section{Resumen}

Objetivo general: Conocer la satisfacción de las usuarias desde la perspectiva del trato recibido durante el embarazo, parto y puerperio, en un hospital público. Método: Estudio descriptivo, prospectivo, transversal. Se elaboró instrumento exprofeso, basado en indicadores de trato digno y dos cuestionarios de satisfacción, piloteado, validado y probada su confiabilidad. Aplicado en puerperio inmediato de parto eutócico. Resultados: 114 encuestadas, análisis bivariado reportó mayor satisfacción en la atención del parto y puerperio en mujeres en unión libre; y menor satisfacción durante el puerperio en usuarias con mayor nivel de escolaridad. Turno nocturno presentó índice de satisfacción más alto en el parto. Discusión: los resultados concuerdan con el «Paradigma de la Desconfirmación», se obtuvo desconfirmación nula (cumpliendo las expectativas), los resultados mostraron buenos índices de satisfacción. Sin embargo, es necesario considerar a las usuarias que expresaron insatisfacción y proponer recomendaciones para mejorar la atención.

Palabras clave: Satisfacción del paciente; trato recibido; embarazo; parto eutócico; puerperio inmediato. 
General Objective: To know about users' satisfaction regarding treatment received during pregnancy, delivery and postpartum at a public hospital. Method: Descriptive, prospective, cross-sectional study. An instrument was deliberately created, based on dignified care indicators and two satisfaction questionnaires, after being piloted, validated and its reliability checked. Applied in immediate postpartum after normal childbirth. Results:114 respondents, bivaried analysis reported more satisfaction in delivery and postpartum care in women in free union, and less satisfaction during postpartum in users with higher levels of education. Night shift presented the highest index of satisfaction in delivery. Discussion: Results agree with the "Disconfirmation Paradigm", null disconfirmation was obtained (fulfilling expectations) and results showed good satisfaction indexes. However, it is necessary to consider the users who expressed dissatisfaction and make recommendations to improve care.

Key words: Patient Satisfaction; treatment received; pregnancy; normal delivery; immediate postpartum.

\section{Resumo}

Objetivo geral: Conhecer a satisfação dos usuários na perspectiva do tratamento recebido durante a gravidez, parto e puerpério, em um hospital público.

Método: Estudo descritivo, prospectivo, transversal. Desenhou-se um instrumento exprofeso, baseado em indicadores de tratamento digno e dois questionários de satisfação, pilotados, validados e checados em sua confiabilidade. Foi aplicado no puerpério imediato de parto eutócico. Resultados: dos 114 entrevistados, a análise bi-variada relatou maior satisfação no atendimento ao parto e puerpério nas mulheres em união livre;e menor satisfação durante o puerpério em usuários com maior nível de escolaridade. A turma da noite apresentou maior índice de satisfação no parto. Discussão: Os resultados concordam com o "Paradigma da Desconfirmação", houve desconfirmación nula (cumprindo as expectativas), os resultados mostraram bons índices de satisfação. No entanto, é necessário considerar os usuários que expressaram insatisfação e propor recomendações para melhorar $\mathrm{o}$ atendimento.

Palavras chave: Satisfação do paciente; tratamento recebido; gravidez; parto eutócico; puerpério imediato.

\section{Introducción}

En México con la finalidad de mejorar la calidad de la atención, a lo largo del tiempo se han llevado a cabo tres reformas en el sistema de salud. La tercera y última reforma, proyectó la cobertura de atención en los servicios de salud(1). En las primeras décadas del siglo XX se inicia la evaluación de la calidad en los servicios de salud, posteriormente, en la segunda mitad de ese siglo esta evaluación se consideró como una herramienta indispensable en la transformación de los sistemas de salud $(2,3)$. En la actualidad como resultado de la tercera reforma, las mujeres que eran atendidas regularmente de manera empírica, han optado por recibir atención prenatal, de parto y del puerperio, por parte de personal perteneciente a instituciones de salud de tipo gubernamental. Los datos publicados por el Instituto Nacional de Estadística y Geografía Informativa (INEGI) reportan que en el período 2004-2009, 97.6\% de mujeres embarazadas recibieron atención de control prenatal y nueve de cada diez fueron atendida por un médico(4).Sin embargo, en el estado de Veracruz la satisfacción en la atención brindada a las usuarias que acuden a los servicios de control prenatal y obstetricia en instituciones del sector salud, no se ha evaluado 
desde la perspectiva del trato recibido y considerando las expectativas de las pacientes. La satisfacción de los usuarios es un componente importante de la calidad de la atención, su importancia radica principalmente en que permite conocer las debilidades de los servicios de salud y actuar para modificar dichas deficiencias, fortaleciendo las expectativas médicas y no médicas del usuario, con respecto al cuidado de su salud. En la evaluación de la satisfacción general se toma en cuenta, el conjunto de juicios del cuidado médico y factores internos del sistema de salud que influyen en la salud del paciente, tales como, la actitud del personal, relación prestador-usuario, la accesibilidad al servicio, la competencia técnica y la eficacia $(5,6)$.Desde este contexto, se consideró importante conocer la satisfacción de la mujer durante el embarazo, parto y puerperio, para identificar los rubros que ameritaran mejorarse y aquellos que fueran necesario evitar. Contemplando que si las usuarias tuvieran una percepción negativa de la atención recibida, podrían optar ser atendidas por parteras no capacitadas, lo que pudiera ocasionar que pongan en riesgo su vida y la de sus hijos. Por lo tanto, se contempló importante el realizar un proyecto de investigación que abordara la satisfacción de la usuaria desde la perspectiva del trato recibido, para llevarlo a cabo en un hospital de segundo nivel de atención perteneciente a los servicios de salud del estado, para conocer la satisfacción de las usuarias durante el embarazo, parto eutócico y puerperio inmediato. Ya que conocer esta situación, proporcionaría información para detectar oportunidades de mejora en el servicio otorgado y de esta manera tener la oportunidad de proponer recomendaciones dirigidas a brindar una atención de calidad, que pudiera incluso, impactar a largo plazo en los índices de morbilidad y mortalidad materna y perinatal. Para la evaluación de los resultados se considero el Paradigma de la Desconfirmación, el cual se basa en la diferencia entre las expectativas ideales y la experiencia que se tiene en un determinado servicio, lo que conlleva a tener un resultado positivo, negativo o nulo de acuerdo a la expectativa previa y a la experiencia vivida al recibir el servicio, de tal forma que el usuario confirma o desconfirma de acuerdo a dicha experiencia. Por lo tanto, se obtiene una desconfirmación positiva si se superan las expectativas, desconfirmación nula en el caso del cumplimiento de las expectativas y desconfirmación negativa cuando no se cumplen las expectativas(7).

El objetivo general del estudio fue: Conocer la satisfacción de las usuarias desde la perspectiva del trato recibido durante el embarazo, parto eutócico y puerperio inmediato, en un hospital de segundo nivel de atención perteneciente a los servicios de salud del estado de Veracruz.

\section{Método}

El tipo de estudio fue observacional, descriptivo, prospectivo y transversal. Se utilizó un muestreo aleatorio simple, el calculo del tamaño de la muestra se realizo considerando el número total de partos eutócicos reportados en el año 2012 (1855 con un promedio de 150 pacientes al mes) en el hospital en donde se llevó a cabo el estudio, quedando constituida por 113 usuarias de acuerdo a la fórmula utilizada. Las pacientes fueron seleccionadas de manera aleatoria considerando el total de mujeres que se encontraban hospitalizadas en el servicio de ginecología y obstetricia en puerperio inmediato por parto eutócico, en los turnos visitados (matutino, vespertino y jornada acumulada)cuidando no duplicar la unidad de estudio. Todas las mujeres incluidas aceptaron participar mediante firma del formato de consentimiento informado. La investigación fue aprobada por el Comité de Ética e Investigación de la institución donde se realizó el estudio, otorgándole el número de registro: JSV/HRLFN/2013/08.El instrumento aplicado fue elaborado exprofeso para el estudio, se le denomino Cuestionario de Satisfacción del Trato Recibido durante el Embarazo, Parto y Puerperio (CUSTREPP). La construcción del mismo se llevó a cabo tomando como referencia indicadores de trato digno, tales como, satisfacción de la información proporcionada por el médico en primer y segundo nivel y, satisfacción por 
el trato recibido en primer y segundo nivel; así como, dos instrumentos para evaluar la satisfacción de la usuaria durante el embarazo, parto y puerperio(8,9). El instrumento quedo estructurado por 6 apartados: a) Datos generales: socio demográficos, ginecoobstetricos, condiciones del RN; b) Atención prenatal ( 7 ítems) con los cuales se obtuvo información del trato recibido al solicitar las consultas, la satisfacción del horario en las mismas y la cantidad de tiempo de espera, importancia y número de exámenes solicitados por parte del médico; c) Atención y competencia profesional (11 ítems) para identificar la satisfacción de la usuaria durante el control prenatal con respecto al trato del personal médico y el personal encargado de la aplicación de las vacunas de toxoide tetánico, así como, la preparación y habilidad para las exploraciones físicas y pruebas solicitadas, por parte del médico; d) Cuidados durante el periodo del parto (11 ítems), que incluyó preguntas para conocer la satisfacción de acuerdo al trato recibido durante la atención del parto por parte de médicos y enfermeras, se incluyó en este apartado un ítem para identificar el turno de atención del parto; e)Cuidados del puerperio inmediato(9 ítems) que proporcionó información acerca del trato recibido en el puerperio inmediato por parte del médico y las enfermeras; f)Aspectos logísticos y derecho a la intimidad (4 ítems) que aportó datos acerca de las características de los alimentos e instalaciones y el respeto a la intimidad por parte del personal de salud. El instrumento fue revisado por expertos y piloteado para obtener su confiabilidad y validez, obteniendo un alfa de Cronbach de 0.934.Las variables consideradas fueron: socio demográficas (edad, ocupación, nivel de estudios, ingresos), gineco obstétricas (menarca, número de embarazos, partos vaginales, cesáreas y abortos), el trato recibido durante el embarazo, parto eutócico y puerperio inmediato, y la satisfacción de la usuaria. Análisis estadístico, al concluir la recopilación de datos se elaboró la base de datos en el software estadístico SPSS versión 19, se realizó el análisis descriptivo el cual se basó en la elaboración de tablas de frecuencia, gráficos de barras y de sectores para las variables cualitativas. Asimismo, para las variables cuantitativas el análisis consistió en medidas de tendencia central y de dispersión, así como gráficos de cajas. Posteriormente se realizó análisis inferencial bivariado, se utilizaronlas pruebas no paramétricas de Kruskal-Wallis y de Mann-Whitney $(\mathrm{p}<0.05)$, además del Coeficiente de correlación de Spearmany se elaboró índice aditivo no ponderado para cada rubro de atención, para contrastar con las variables socio demográficas y gineco obstétricas.

\section{Resultados}

Se entrevistaron 114 usuarias, la media de edad fue 24.3 años, la menor de 15 años y la mayor de 41. El 25\% de las mujeres entrevistadas se encontraban entre los 15 y los 20 años de edad; $50 \%$ de las pacientes tenían 23 años o menos. Estado civil, $17.5 \%$ casadas, $65.8 \%$ unión libre, $84.2 \%$ con pareja estable y $15.8 \%$ sin pareja estable; ocupación, $84.2 \%$ amas de casa, $7 \%$ empleadas y $7.9 \%$ estudiantes; escolaridad, $22.8 \%$ con secundaria completa, $21.9 \%$ con primaria completa, $19.3 \%$ bachillerato completo, $9.6 \%$ primaria incompleta y bachillerato incompleto $9.6 \%$, secundaria incompleta $5.3 \%$, universidad incompleta $5.3 \%$, universidad completa $3.5 \%$ y sin estudios $2.6 \%$. El $50 \%$ de las pacientes reportó un ingreso mensual de entre 0 y 2,699 pesos mexicanos y $47.4 \%$ su ingreso se encontraba entre $\$ 2,700$ y $\$ 6,799$ pesos mexicanos. Antecedentes gineco-obstétricos, $36.85 \%$ con menarcaa los 12 años; $36.8 \%$ era primigesta, $27.2 \%$ dos gestaciones, $14.9 \%$ tres gestaciones, y $10.5 \%$ cuatro gestaciones. Partos reportados, $44.7 \%$ un parto, $24.6 \%$ dos partos, $14.9 \%$ tres partos y $11.4 \%$ cuatro partos. En cuanto a las cesáreas, $92.1 \%$ dijo que no se las han realizado y al $86.8 \%$ no se le han practicado abortos.

El 95.6\% la atendió un médico durante el control prenatal y 13.2\% personal de enfermería; $19.3 \%$ presentó complicaciones prenatales $(15.8 \%$ preclampsia, $26.3 \%$ amenaza de aborto, $47.4 \%$ infección de vías urinarias y $10.5 \%$ vulvo vaginitis). La atención del parto fue realizada por un médico en $89.5 \%, 21.1 \%$ por un estudiante y $19.3 \%$ por personal de 
enfermería; $11.4 \%$ presentó complicaciones durante el parto (13.3\% hipoxia del recién nacido, $20 \%$ óbito fetal, $6.7 \%$ parto pretérmino, $13.3 \%$ parto pos término, $13.3 \%$ distocia de trabajo de parto, $13.3 \%$ parto fortuito, $6.7 \%$ hemorragia, $6.7 \%$ plaquetopenia y $6.7 \%$ desgarro perineal). Durante el puerperio, $80.7 \%$ fue atendida por personal de enfermería, $59.6 \%$ por un médico y $12.3 \%$ por un estudiante; $94.7 \%$ no presentó complicaciones durante el puerperio y $5.3 \%$ si las presentó $(16.7 \%$ anemia, $50 \%$ fiebre, $16.7 \%$ tenesmo vesical y $16.7 \%$ reacción alérgica a medicamento). De las complicaciones encontradas durante el embarazo, parto eutócico y puerperio inmediato, las que presentaron mayor frecuencia fueron: en el embarazo la infección de vías urinarias, durante el parto eutócico el óbito fetal y en el puerperio inmediato la fiebre. En relación a las consultas del seguimiento durante el embarazo (control prenatal), el mayor porcentaje fue de 6 consultas (20.2\%), $19.3 \% 8$ consultas; y $14 \% 5$ y 7 consultas. El 55\% de las usuarias no emitió ningún comentario extra respecto de la tención recibida, $23 \%$ mencionó comentarios negativos y $22 \%$ hizo comentarios positivos. Comentarios negativos expresados, $34.6 \%$ mal trato por parte del personal de enfermería, $19.2 \%$ falta de personal, $15.4 \%$ mal trato por parte de los médicos, $7.7 \%$ tiempo de espera prolongado en el servicio de urgencias, $3.8 \%$ mal estado y deficiencia de ropa de cama, 3.8\% poco número de camas, $3.8 \%$ malas instalaciones, $3.8 \%$ poco tiempo durante la visita, $3.8 \%$ mal trato por parte del personal de trabajo social, $3.8 \%$ mal trato del personal de seguridad.

Para llevar a cabo el análisis bivariado se realizaron las pruebas no paramétricas de Kruskal-Wallis y de Mann-Whitney ( $<<0.05)$, además del Coeficiente de correlación de Spearman. Se calcularon índices aditivos para cada una de las tres áreas de atención. Los índices consistieron en totalizar la calificación que dio cada paciente para las preguntas correspondientes a cada área. El índice reflejaba que a mayor valor, mayor será la calificación de satisfacción que la paciente otorga.

El cuadro I presenta los datos sobre la relación entre la satisfacción de la usuaria y las variables socio demográficas y económicas de la paciente, se observó mayor satisfacción en la atención del parto en mujeres en unión libre y menor satisfacción durante el puerperio en usuarias con mayor nivel de escolaridad. Cuadro II muestra el índice de satisfacción durante la atención en el período de parto por turno, el Turno nocturno presentó el índice de satisfacción más alto durante el parto.La relación entre la satisfacción de la usuaria y las complicaciones durante el embarazo, parto y puerperio inmediato se muestra en el cuadro III. En el cuadro IV se presenta la relación entre la satisfacción de la usuaria y el estado del recién nacido. La correlación entre la satisfacción de la usuaria durante el embarazo, parto y puerperio en relación al número de embarazos, partos y abortos se muestra en el cuadro V. La edad de la paciente no mostró una correlación significativa en relación a la satisfacción durante el embarazo, parto y puerperio.

Cuadro I. Relación entre el puntaje de satisfacción por área de atención y variables demográficas y económicas de la paciente

\begin{tabular}{rrrrr}
\hline \multicolumn{1}{c|}{ Variable } & Embarazo & \multicolumn{1}{c|}{ Parto } & Puerperio \\
\hline \multicolumn{1}{c}{ Estado Civil } & $\mathbf{7 5 . 1}$ & $\mathbf{4 7 . 7}$ & $\mathbf{5 4 . 8}$ \\
Casada & 74.6 & 44.5 & 51.7 \\
Unión libre & 76.2 & 48.96 & 55.9 \\
Otra & 71.7 & 46.2 & 54.0 \\
P-value & 0.273 & $\mathbf{0 . 0 3 2 ^ { * }}$ & 0.108 \\
\hline Pareja Estable & $\mathbf{7 5 . 1}$ & $\mathbf{4 7 . 7}$ & $\mathbf{5 4 . 8}$ \\
& $\mathrm{Si}$ & 75.8 & 48.0 & 55.0 \\
$\mathrm{No}$ & 71.7 & 46.1 & 54.16 \\
P-value & 0.180 & 0.308 & 0.691 \\
\hline \multicolumn{2}{|c|}{ Ocupación } & $\mathbf{7 5 . 1}$ & $\mathbf{4 7 . 7}$ & $\mathbf{5 4 . 8}$ \\
Ama de casa & 75.6 & 47.6 & 55.1 \\
Empleada & 73.2 & 47.8 & 55.0 \\
Estudiante & 71.8 & 47.8 & 51.7 \\
P-value & 0.582 & 0.994 & 0.501
\end{tabular}




\begin{tabular}{rrrr}
\hline Escolaridad & $\mathbf{7 5 . 1}$ & $\mathbf{4 7 . 7}$ & $\mathbf{5 4 . 8}$ \\
Sin escolaridad & 75.3 & 50.0 & 60.6 \\
Primaria & 77.3 & 48.0 & 57.7 \\
Secundaria & 74.2 & 47.7 & 54.5 \\
Bachillerato & 74.6 & 46.8 & 52.3 \\
Universidad & 72.2 & 48.6 & 52.0 \\
P-value & 0.731 & 0.913 & $\mathbf{0 . 0 3 5}$ \\
\hline Ingreso & $\mathbf{7 5 . 1}$ & $\mathbf{4 7 . 7}$ & $\mathbf{5 4 . 8}$ \\
$(0-\$ 2,699)$ E & 76.2 & 48.1 & 55.2 \\
$(\$ 2,700-\$ 6,799)$ D & 74.2 & 47.2 & 54.4 \\
(\$6,800-\$11,599)D+ & 71.0 & 49.6 & 55.3 \\
P-value & 0.567 & 0.724 & 0.879 \\
\hline *Prueba no paramétrica de Kruskal-Wallis & \\
\hline
\end{tabular}

Fuente: CUSTREPP (mayo-octubre 2013)

Cuadro II. Relación entre la satisfacción y el turno de atención del parto

\begin{tabular}{c|c|c}
\hline Turno & Media & Des. Est. \\
\hline Matutino & 44.45 & 8.99 \\
Vespertino & 46.95 & 6.53 \\
Nocturno & $\mathbf{4 9 . 5 8}$ & 5.96 \\
Total & $\mathbf{4 7 . 8 3}$ & $\mathbf{7 . 3 1}$ \\
\hline
\end{tabular}

Prueba no paramétrica de Kruskal-Wallis

Fuente: CUSTREPP (mayo-octubre 2013)

Cuadro III. Relación entre la satisfacción y las complicaciones durante el embarazo, parto y puerperio inmediato

\begin{tabular}{l|cr|r}
\hline & \multicolumn{2}{|c|}{ Complicaciones } & \multirow{2}{*}{ P-value * } \\
\cline { 1 - 3 } Etapa de atención & Si (\%) & No (\%) & \\
\hline Etapa de control prenatal & $74.27(72.5)$ & $75.40(76.0)$ & 0.3443 \\
Etapa de parto & $45.38(49.0)$ & $48.03(49.0)$ & 0.6204 \\
Etapa de puerperio & $53.33(52.5)$ & $54.96(55.5)$ & 0.4996 \\
\hline
\end{tabular}

* Prueba no paramétrica de Mann-Whitney para muestras independientes.

Fuente: CUSTREPP (mayo-octubre 2013)

Cuadro IV. Relación entre la satisfacción y el estado del recién nacido

\begin{tabular}{l|c|c|r}
\hline & \multicolumn{1}{|c|}{ Estado del recién nacido } & \multirow{2}{*}{ P-value * } \\
\cline { 1 - 3 } \multicolumn{1}{c|}{ Etapa de atención } & \multicolumn{1}{c}{ Vivo } & \multicolumn{1}{c}{ Muerto } & \\
\hline Atención durante el embarazo & $\mathbf{7 5 . 4 5 ( 1 1 . 9 )}$ & $\mathbf{6 7 . 7 5}(\mathbf{7 . 1 4 )}$ & $\mathbf{0 . 0 9 2 7}$ \\
Atención durante el parto & $47.83(7.22)$ & $45.00(10.23)$ & 0.4790 \\
Atención durante el puerperio & $54.86(8.18)$ & $55.25(10.44)$ & 0.8650 \\
\hline
\end{tabular}

* Prueba no paramétrica de Mann-Whitney para muestras independientes.

Fuente: CUSTREPP (mayo-octubre 2013)

Cuadro V. Correlación entre satisfacción por área de atención en relación al número de embarazos, partos y abortos

\begin{tabular}{lcc|c}
\hline & Embarazo & Parto & Puerperio \\
\hline Número de partos & $-0.0390(0.6801)$ & $0.0411(0.6644)$ & $0.1025(0.2779)$ \\
Número de embarazos & $0.0257(0.7857)$ & $0.0759(0.4224)$ & $0.0869(0.3578)$ \\
Número de abortos & $0.0677(0.4741)$ & $0.1017(0.2816)$ & $0.0105(0.9116)$ \\
\hline
\end{tabular}

Coeficiente de correlación de Spearman (P-value) 
La satisfacción del usuario es un indicador de calidad de la atención, la cual se centra en cubrir las necesidades en salud de la población, así como sus expectativas. La evaluación de la satisfacción del usuario es primordial como garantía de calidad y generalmente se lleva a cabo mediante instrumentos proporcionados a los usuarios. La satisfacción del usuario es multifactorial, en ella convergen diversos factores que pueden ser internos y externos a los servicios de salud. En la satisfacción con la atención recibida pueden influir, la edad, el sexo, el nivel socioeconómico, la escolaridad, la ocupación, las patologías psiquiátricas y la relación con los prestadores del servicio, entre otros factores.

La importancia de evaluar la satisfacción del paciente, se centra en detectar oportunidades de mejora y proponer alternativas, con la finalidad de cubrir las expectativas del usuario. La participación de la sociedad al emitir su opinión sobre los servicios que recibe, favorece el empoderamiento de los usuarios para requerir que se cumplan sus perspectivas.

En el presente estudio se pudo observar que el promedio de edad de las mujeres entrevistadas durante el puerperio inmediato fue de 24 años, lo que contrasta con el estudio realizado por Maderuelo (2006) en España(64), quien detectó un promedio de edad de 30 años. En cuanto a la ocupación se observó que la mayoría de las pacientes entrevistadas (84.2\%) se dedicaban a las labores del hogar, lo que coincide con el estudio llevado a cabo por García (2009) en México(65). El 65.8\% de las usuarias entrevistadas se encontraban en unión libre y $17.5 \%$ casadas, datos que difieren con los encontrados por Maderuelo (64) los cuales mostraron que $85.7 \%$ de las usuarias entrevistadas estaban casadas, sin embargo, los porcentajes coinciden en cuanto a que la mayoría de la población estudiada contaba con pareja estable.

En esta investigación $44.7 \%$ de las mujeres incluidas en la muestra expresaron ser primíparas, datos que discrepan con el estudio realizado por Aramburú (2008) en Perú(66) en un pequeño porcentaje, ya que este autor reportó que del total de las usuarias entrevistadas, $53 \%$ fue primípara.

En cuanto al promedio de ingreso mensual, en el presente estudio se observó que 50\% de las usuarias tienen como promedio de ingreso mensual en su hogar, $\$ 0$ a $\$ 2,699$ y $47.4 \%$ de $\$ 2,700$ a $\$ 6,799$. Además, los resultados del análisis bivariado no mostraron relación entre la satisfacción y el ingreso económico, caso contrario al estudio realizado por Ortiz (2003) en México(67), quien reportó asociación entre esta variable y la satisfacción, manifestando que a menor ingreso económico, menor el nivel de exigencia y por lo tanto mayor el nivel de satisfacción.

En relación a las principales complicaciones encontradas durante el embarazo, parto y puerperio inmediato las más frecuentes fueron, infección de vías urinarias durante el embarazo, óbito fetal durante el parto y fiebre durante el puerperio inmediato. A pesar de que en el presente estudio no se valoró la calidad técnica, como tal, a través de las complicaciones se puede inferir que existen casos de atención probablemente inadecuada al encontrarse 3 óbitos fetales, 2 partos fortuitos, 2 partos postérmino, 1 desgarro perineal y 3 cuadros febriles durante el puerperio inmediato. Considerando, además, que la mayoría de las usuarias obtuvieron 6 consultas prenatales, mismas que bien pueden servir para llevar a cabo medidas preventivas y de diagnostico oportuno parar éstas complicaciones. El análisis de los datos obtenidos arrojó que el área de oportunidad que presentó mayor frecuencia, fue el maltrato por parte del personal de enfermería, situación que coincide con el estudio que realizó Nava(2011) en México(10), quien expresó que una de las principales áreas de oportunidad es fortalecer la percepción del trato digno por parte del personal de enfermería, este autor señala que la carencia de trato digno por parte del personal es multifactorial.

Se podría pensar que probablemente las usuarias detectaron con mayor facilidad la carencia de trato digno por parte del personal de enfermería, al estar en mayor contacto 
con dicho personal, ya que las usuarias pasan mayor tiempo con este tipo de personal en comparación con el resto de los prestadores de servicio, las pacientes entrevistadas, $80.7 \%$ refirieron haber sido atendidas por personal de enfermería durante el puerperio inmediato. Cabe mencionar que 55\% de las entrevistadas no emitió ningún comentario, $26 \%$ emitieron alguna oportunidad de mejora y $25 \%$ emitió algún comentario positivo, con éstos datos podrían pensarse que posiblemente las usuarias que no emitieron ningún comentario se sintieron insatisfechas en algún aspecto de la atención, sin embargo, prefirieron no manifestar su probable insatisfacción por temor a tener represalias, a pesar de que en el momento de ser entrevistadas se les comentó en repetidas ocasiones que la información era totalmente confidencial y anónima. Estos datos contrastan con los encontrados por Vicuña (2002)(68), quien manifestó que 92.5\% de las pacientes emitieron alguna queja.

Con respecto a la relación entre el estado civil y la satisfacción, en el presente estudio se pudo observar que las pacientes que viven en unión libre presentaron mayor satisfacción, en comparación con las usuarias casadas. Situación que se podría atribuir a que durante la aplicación del instrumento, las parejas de las mujeres casadas mostraron mayor interés por que se les proporcionara atención con calidad, a diferencia de las parejas de las mujeres en unión libre que estuvieron presentes durante la aplicación del instrumento, quienes no comentaron nada sobre la atención que habían recibido. Por lo que se piensa, que las mujeres casadas pudieron estar influenciadas por las expectativas de su pareja.

En cuanto a la atención durante el puerperio, ésta mostró la calificación más alta en relación a la satisfacción por parte de las usuarias que no cuentan con escolaridad, y en éste mismo rubro la calificación más baja de satisfacción la manifestaron las pacientes con escolaridad de bachillerato o universidad. Estos resultados son similares a los mostrados por Ortiz(2003) (67), quien reportó que la satisfacción tiene relación directamente proporcional con la escolaridad del usuario, es decir a mayor escolaridad menor satisfacción y viceversa.

La satisfacción por parte de la usuaria en relación al turno en que fue atendido el parto, mostró que el turno nocturno presentó datos de mayor satisfacción, esto podría ser atribuido a las características individuales del personal de dicho turno o a la dinámica del mismo. Considerando que en el turno nocturno probablemente se realizan menos actividades administrativas que en los turnos matutino y vespertino, lo que da la oportunidad al personal de disponer de mayor tiempo para brindar la atención a los pacientes y esto podría ser un condicionante para que las usuarias expresaran una mejor satisfacción.

En este estudio se pudo observar que la satisfacción durante el embarazo, parto y puerperio inmediato en relación con las complicaciones, no presentó diferencias significativas. Caso contrario a lo encontrado en el estudio realizado por Maderuelo (64) el cual reportó asociación entre la ausencia de complicaciones, con una mayor satisfacción. Igualmente, el hecho de que estuviera vivo o muerto el recién nacido no fue un factor que se asociara a la satisfacción de la madre durante el parto y puerperio inmediato. Caso contrario se presentó durante la atención al embarazo, probablemente el hecho de que el $\mathrm{RN}$ falleciera determinó la satisfacción de las pacientes en este periodo, ya que el instrumento de las tres áreas de atención, fue aplicado durante el puerperio inmediato y probablemente el fallecimiento del RN hizo reflexionar a la usuaria, que quizá el control prenatal fue llevado de manera inadecuada y por tal motivo se originó la muerte del RN.

Finalmente se pudo observar que no se presentó relación estadísticamente significativa entre la edad de las pacientes y la satisfacción de las mismas durante el control prenatal, parto y puerperio, situación que coincide con el estudio que realizó Maderuelo (64).

El análisis general de la presente investigación reporta que la mayoría de las pacientes manifestaron un buen nivel de satisfacción con respecto al trato recibido, sin embargo, se puede comentar que los resultados se pueden relacionar con la teoría del Paradigma de la Desconfirmación. En la cual, la satisfacción depende de las expectativas que se tienen de acuerdo a la experiencia del usuario para determinar la calidad del servicio y los resultados obtenidos. Por lo tanto, se tiene una desconfirmación positiva si se superan 
las expectativas, desconfirmación nula en el caso del cumplimiento de las expectativas y desconfirmación negativa cuando no se cumplen las expectativas. De tal forma, se podría manifestar que los resultados obtenidos en este estudio muestran una desconfirmación nula, ya que de acuerdo a la satisfacción expresada por las pacientes se cumple con las expectativas esperadas. Sin embargo, aquí habría que considerar que las usuarias que se manifestaron satisfechas, podrían cambiar de opinión al reflexionar posteriormente sobre el trato recibido, una vez libres de la emoción del evento, ya que la satisfacción puede estar influenciada por la emoción que se vive, en el momento que se le pide su opinión.

Asimismo, es importante señalar que considerando las características de la población estudiada (población abierta que pertenece al SP y la cual difícilmente ha tenido acceso a una atención en otros niveles) la percepción de trato digno recibido y sus expectativas pudieran ser limitadas, además de considerar que este tipo de población frecuentemente ignora los derechos que como paciente tiene.

A pesar de que los resultados mostraron que en la mayoría de las usuarias la satisfacción es favorable, se debería tomar en cuenta que también existieron opiniones de insatisfacción respecto de la atención recibida, las cuales se deben de tomar en cuenta como oportunidades de mejora, con la finalidad de mejorar la calidad de la atención en la prestación de servicios. Evitando así, que las usuarias que se manifestaron insatisfechas obtén por no regresar a dichas instituciones de salud para recibir atención en próximas intervenciones y puedan con esto, poner incluso en peligro su vida y la de sus hijos al preferir recibir atención por parte de personal empírico.

\section{Bibliografía}

1. Organización Mundial de la Salud (OMS) Informe sobre la salud en el mundo 2000. Mejorar el desempeño de los sistemas de salud.Ginebra, Suiza 2000.

2. Ramírez STJ, Nájera AP, Nigenda LG. Percepción de la calidad de la atención de los servicios de salud en México: perspectiva de los usuarios. SaludPublicaMex 1998;40:3-12.

3. Rahmqvist M. Patient satisfaction in relation to age, health status and Dther background factors:A model for comparisons of care units. Int I Quality Health Care 2001:13(5):385-390.

4. Instituto Nacional de Estadística y Geografía (INEGI).Estadísticas a propósito del día de la mujer. Datos México, 2012.

5. Oliva C, Hidalgo C. Satisfacción usuaria: un indicador de calidad del modelo de salud familiar, evaluada en un programa de atención de familias en riesgo biopsicosocial en la atención primaria. Psykhe 2004; 13(002): 173-186

6. Donoso N, Díaz M, Peralta J, López C, Garrido S. Medición nacional de satisfacción usuaria en la red pública de salud de chile, Aplicación 2009.Chile: Departamento de participación social y trato al usuario, Subsecretaría de Redes Asistenciales.2009

7. Moliner B. Berenguer G. Gil I. La importancia de la preformance y las expectativas en la formación del consumidor. Investigaciones Europeas 2001; 7(3):155-172

8. Vivanco M. Análisis de la satisfacción de las madres durante el trabajo de parto y puerperio inmediato en un hospital público de tercer nivel de la comunidad de Madrid. Validación y adaptación transcultural de la escala de satisfacción COMFORTS. Reduca. 2009; 1(2):275-299

9. Maderuelo J. Haro A. Pérez F. Cercas L. Valentín A. Moran E. Satisfacción de las mujeres con el seguimiento del embarazo. Diferencias entre los dispositivos asistenciales. GacSanit. 2006; 20(1):31-9

10. Nava M, Brito J, Valdez A, Juárez A. Trato digno otorgado por enfermeras en centros de salud en Morelos, México. RevEnfermInstMex Seguro Soc. 2011; 19 (2): 91-98

11. Sainz M. Sánchez Y. Granados G. Encuesta de satisfacción en embarazadas captadas 
en Atención Primaria. Rev el trabajo en nuestros centros.2001:506-510.

12. Seclen-Palacín JA, Benavides B, Jacoby E, Velásquez A, Watanabe E. ¿Existe una relación entre los programas de mejora de la calidad y la satisfacción de usuarias de atención prenatal?: experiencia en hospitales del Perú. Rev Panam Salud Pública. 2004; 16(3):149-57.

13. Villanueva A, Riega P, Torres J, Yabar C, Alarco O. Cumplimiento de Estándares de Calidad en la Atención del Parto Institucional y Nivel de Satisfacción de Usuarias. Ciencia e investigación Médica Estudiantil Latinoamericana.2003;8 (1):13-18

14. Valderrama S. Percepciones y satisfacción de la mujer rural en la atención del parto institucional y tradicional en la provincia Sánchez Carrión .Rev Salud, sexualidad, y sociedad 2008; 3(1,2)

15. Ramírez O, López A, Barragán A, Arce E.Satisfacción del usuario en una unidad de medicina familiar de una institución de seguridad social de la ciudad de México. Archivos en Medicina Familiar.2005; 7(1):22-26

16. Guzmán M, Ramos L, Castañeda O, López D, Gómez A. Satisfacción de los usuarios de una unidad médica familiar. Rev Med Inst Mex Seguro Soc 2006; 44 (1): $39-45$

17. Velázquez L, Ruiz N, Trejo A, Sotelo J. La satisfacción de los usuarios en relación a la calidad de atención en el Instituto Nacional de Neurología y Neurocirugía. Medigraphic.2006; 11(3):177-184

18. Puentes Rosas E, Gómez Dantés O, Garrido Latorre F. Trato a los usuarios en los servicios públicos de salud en México. RevPanam Salud Pública. 2006; 19(6):394402

19. Ávila L, Wirtz V, Serván E, Bautista S, Barroso C, González M. Utilización de servicios de salud. Encuesta Nacional de Salud y Nutrición. Resultados Nacionales 2012. 2012:42-54

20. Pérez R, Doubova SV, Wirtz VJ, Servan-E, Dreser A, Hernandez.BMJ Open. 2014; 4(5): e004669

21. Reyes H. Flores S. Sauceda A. Vertiz R. Juárez C. Wirtz V.et al. Percepción de los usuarios sobre la calidad de la atención ambulatoria en servicios de salud en México Rev. salud pública de Mexico.2013; 55 supl 2:S100-S105

22. Landa F. Méndez F. Muñoz M. Satisfacción de los usuarios con los servicios de estomatología en el IMSS. RevMedInstMex Seguro Soc 2007; 45 (2): 149-155

23. Frenk J, Gómez O. Para entender el sistema de salud en México. México. Nostra ediciones. 2008

24. Parker S, Rubalcava L, Identificación y análisis de los efectos en las condiciones de salud de los afiliados al Seguro Popular. Centro de investigación y Docencia Económicas A.C.2006; 1-78

25. Lusting N, Políticas públicas y salud en México, Rev nexos 2008; 367:3-27

26. Guajardo E. Administración de la calidad total; conceptos y enseñanzas de los grandes maestros de la calidad. México. Editorial Pax México.2003

27. Van Dick J. Bases conceptuales de la calidad de atención a la salud. Cuaderno de divulgación científica No 29 México.2001

28. Rosas C. Evaluación de la calidad del desempeño del personal de enfermería en las actividades prioritarias en el primer nivel de atención. Gestión del conocimiento III y salud pública III: productos de los módulos. 2008:95-128

29. Donabedian A. Garantía y monitoria de la calidad de la atención medica. México. Topografía Fenian.1990

30. Donabedian A. La calidad de la atención médica; Definición y métodos de evaluación. México ediciones copilco, S.A. De C.V.; 1991

31. NORMA Oficial Mexicana NOM-007-SSA2-1993, Atención de la mujer durante el embarazo, parto y puerperio y del recién nacido. Criterios y procedimientos para la 
prestación del servicio

32. Rigol. Obstetricia y Ginecología. La Habana. Editorial Ciencias Médicas.2004.

33. Lazcano-Ponce E, Schiavon R, Uribe-Zuñiga P, Walker D, Suárez-López L, LunaGordillo R, Ulloa-Aguirre A. Cobertura de atención del parto en México. Su interpretación en el contexto de la mortalidad materna. Salud Pública Mex 2013; 55 supl 2: S214-S224.

34. Heredia-Pi I, Serván-Mori E, Reyes-Morales H, Lozano R. Brechas en la cobertura de atención continua del embarazo y el parto en México. Salud Pública Mex 2013; 55 supl 2:S249-S258

35. Comité internacional de bioética, UNESCO. Bioética: en busca de consensos; Proyecto de informe sobre responsabilidad social y salud. México. El colegio Nacional.2009:18-21

36. Organización Panamericana de la Salud. La Salud Pública en las Américas. Nuevos Conceptos, Análisis del Desempeño y Bases para la Acción. Washington, D.C.: OPS.2002

37. Serrano-del Rosal R, Loriente-ArinN. Theanatomy of patientsatisfaction. Salud Pública Mex. 2008; 50:162-172

38. Bobadilla J. Observatorio de la Salud, Políticas de la salud: La Reforma en México y en el Mundo.1996:255-400.

39. Sofaer S. FirmingerK.Patient perceptions of the quality of health services.Rev. Public Health 2005.26:513-59

40. Carmona L. Leal M. La teoría de los dos factores en la satisfacción del cliente. Investigaciones Europeas, Vol. 4, N 1, 1998; 4: 53-80

41. Mira JJ, Aranaz J. La satisfacción del paciente como una medida del resultado de la atención sanitaria. Medicina Clínica 2000; 114 (Supl 3):26-33

42. Oliva C, Hidalgo C. Satisfacción usuaria: un indicador de calidad del modelo de salud familiar, evaluada en un programa de atención de familias en riesgo biopsicosocial en la atención primaria. Psykhe 2004; 13(002): 173-186

43. Jiménez R. Indicadores de calidad y eficiencia de los servicios hospitalarios. Una mirada actual. Rev Cubana Salud Pública 2004; 30(1):17-36

44. Donoso N, Díaz M, Peralta J, López C, Garrido S. Medición nacional de satisfacción usuaria en la red pública de salud de chile, Aplicación 2009.Chile: Departamento de participación social y trato al usuario, subsecretaría de redes asistenciales.2009

45. Frenk J, Gómez O. Bioética en acción: fundamentos éticos de las políticas públicas en salud. Memorias CNB Aspectos sociales de la bioética. México 2010:77-78

46. Echeverri M. Derecho a la salud, estado y globalización. RevFacNac Salud Pública 2006; 24 número especial: 80-95

47. Ley General de Salud. Últimas reformas publicadas DOF 15-01-2014

48. Ruelas E, Reyes H, Zurita B, Vidal L, Karchmer S, Círculos de calidad como estrategia de un programa de garantía de calidad de la atención médica en el Instituto Nacional de Perinatología. Salud Pública Mex1990; 32:207-220

49. Aguirre H. Evaluación de la calidad de la atención medica. Expectativas de los pacientes y de los trabajadores de las unidades médicas. Salud Pública Mex 1990; 32:170-180

50. Ruiz M, Martínez G, Calvo J, Aguirre H, Arango R, Lara R, Camino M, et al. Bases para la evaluación de la calidad de la atención en las unidades médicas del sector salud, Salud Publica Mex 1990; 32:156-169

51. Tapia J, Urdapilleta O, Gómez O, Garrido F, Evaluación del gasto en la construcción de unidades de salud: ejemplo de participación comunitaria. Salud Pública Mex 2003; 45:483-491

52. Garmendia A, Nava F. Los trabajadores en la estrategia de calidad del IMSS. El cotidiano 2002; 18 (112):15-23 
53. Programa de Mejoría Continua de la Calidad de la Atención Médica. Secretaria de Salud. México

54. Secretaria de Salud. Programa Nacional de Salud 2001-2006, Programa de acción: Cruzada Nacional por la Calidad de los Servicios de Salud. México

55. Programa de acción específico 2007-2012. Arranque parejo en la vida

56. Embarazo Saludable/ Seguro Médico para una Nueva Generación. Seguro popular. 2012 México

57. Programa de Acción Especifico 2007-2012 Sistema Integral de Calidad en Salud SICALIDAD. México

58. Programa de calidad efectiva en servicios de salud PROCESS 20132018. México

59. Pelcastre B. Villegas N. De León V. Díaz A. Ortega D. Santillana M.et al. Embarazo, parto y puerperio: Creencias y prácticas de parteras en San Luis Potosí, México. RevEscEnferm USP 2005; 39(4):375-82.

60. ZukangS.Ban K. Panorama general .Objetivos de desarrollo del milenio informe 2012.2012:1-5

61. Bruno J, Uría U, García M, Suárez D, MusleraCanclini, E, Álvarez G. Atención maternal y neonatal. I Encuesta de opinión. Consejería de Salud y Servicios Sanitarios. Principado de Asturias. 2010.www. astursalud.es.

62. Tataje $\mathrm{O}$. Tesis nivel de satisfacción de la puérpera sobre la atención que brinda la enfermera en el hospital nacional docente madre niño San Bartolomé, noviembre 2003. Universidad Nacional de San Marcos. Lima. 2004

63. Vivanco M. Análisis de la satisfacción de las madres durante el trabajo de parto y puerperio inmediato en un hospital público de tercer nivel de la comunidad de Madrid. Validación y adaptación transcultural de la escala de satisfacción COMFORTS. Reduca. 2009; 1(2):275-299

64. Maderuelo J. Haro A. Pérez F. Cercas L. Valentín A. Moran E. Satisfacción de las mujeres con el seguimiento del embarazo. Diferencias entre los dispositivos asistenciales. GacSanit. 2006; 20(1):31-9

65. García J. Medina S. Factores asociados a la satisfacción materna con la experiencia del nacimiento de su hijo. Bol Clin Hosp Infant Edo Son 2009; 26(1): 23-27

66. Aramburú P. Arellano R. Jauregui S. Pari L. Salazar P. Sierra Oswaldo. Prevalencia y factores asociados a depresión posparto en mujeres atendidas en establecimientos de salud de primer nivel de atención en Lima Metropolitana, junio 2004. Rev. Perú. Epidemiol.2008; 12 (3): $1-5$

67. Ortiz R. Muñoz S. Lechuga D. Torres E. Consulta externa en instituciones de salud de Hidalgo, México, según la opinión de los usuarios. Rev Panam Salud Pública/Pan Am J Public Health. 2003; 13(4):229-238

68. Vicuña M. Nivel de satisfacción y disfunciones percibidas sobre la calidad de atención de los servicios obstétricos.Anales de la Facultad de Medicina Universidad Nacional Mayor de San Marcos.2002; 63(1): 40-50

69. Declaración de Helsinki de la Asociación Médica Mundial.

70. Reglamento de la Ley General de Salud en Materia de Investigación para la Salud 